\title{
Intra-operative Sensemaking by Surgeons: Analysis of Operative Reports for Uncertainty and Irregular Cues
}

\author{
Lygia Stewart \\ University of California, \\ San Francisco \\ lygia.stewart@va.gov
}

\author{
Cynthia O. Dominguez \\ Klein Associates, a Division of ARA \\ cindy@decisionmaking.com
}

\author{
Lawrence W. Way \\ University of California, \\ San Francisco \\ lwway@aol.com
}

\begin{abstract}
Motivation - Understanding surgeons' intraoperative sensemaking and whether linguistic and descriptive choices reflect unconscious thought. Research approach - Analysis of surgeons' sensemaking, using laparoscopic cholecystectomy operative reports of cases with and without bile duct injuries, examining for linguistic modifiers of uncertainty and description of irregular cues. Findings/Design - qualifiers of uncertainty and an increased number of irregular cues were more common in bile duct injury cases. Research Implications - These data suggest the unconscious mind detects aberrations whose significance cannot break through to conscious recognition. Originality/Value - Both complicated and uncomplicated cases were studied. The importance of linguistic qualifiers has not been previously studied in this domain. Take away message - descriptive and linguistic choices reflect unconscious thought.
\end{abstract}

\section{Keywords}

Sensemaking, Surgery, linguistics.

\section{INTRODUCTION}

Laparoscopic cholecystectomy has an excellent safety record. The most serious, although rare, complication is bile duct injury. We previously reported that this stems from a visual illusion whereby the common bile duct appears to be the cystic duct. If the illusory nature of the anatomic premise is not detected, the wrong duct is cut. The illusion is created from the normal heuristics of visual perception, and detection is hindered by the loss of haptic input inherent in the laparoscopic method. (Way et al 2003, Stewart \& Way 2007) We also studied the surgeons' sensemaking processes, and recognized the importance of being able to re-frame the original anatomic premise as a first step to dispel the illusion. (Stewart et al 2007) The language of certainty or uncertainty may reflect unconscious assessments of the surgeon's confidence in the operative dissection. We examined cases with and without biliary injuries, whether detected or not by the surgeon during the operation. We compared these reflections of the surgeons' sensemaking during noncomplicated versus complicated laparoscopic cholecystectomies.

\section{METHODS}

The operative report, a verbal description of the case, is dictated by the surgeon immediately after the operation, but usually before technical complications have been diagnosed (unless the injury is diagnosed intra-operatively). It is the most objective and accurate record of the surgeon's perception of the case. We analyzed 250 laparoscopic cholecystectomy operative notes from 153 surgeons. 125 operations were uncomplicated and 125 had associated bile duct injuries (BDI). The text of the operative reports was entered into a database of key elements of the case. No attempt was made to read between the lines; the surgeon's words were the data analyzed. Operative reports were queried for the presence and number of irregular cues (abnormal or additional arteries, veins, bile ducts, cholangiography, etc.). The exact description and the overall number of irregular cues was analyzed.

Semantic choices were studied as insights into cognition. The text of the operative report was queried for linguistic qualifiers of certainty (clear, positive, sure, certain, confident, agreement, easily, obvious, without difficulty) or uncertainty (felt, appear, thought) during dissection of the cystic duct. Using this analysis the operative reports fell into three distinct groups: those with qualifiers of certainty (certainty), those with qualifiers of uncertainty (uncertainty), and those with no qualifiers. Three groups were analyzed: 1) uncomplicated cases, 2) cases with BDI undetected by the surgeon (unrecognized BDI), and 3) cases with BDI that were detected by the surgeon intra-op (recognized BDI).

\section{RESULTS}

Fewer irregular cues were reported in uncomplicated cases than in those with a BDI ( 0.44 vs 2.3 , uncomplicated vs BDI, $\mathrm{P}<0.0001)$. Surgeons also reported more irregular cues in cases with intra-op BDI recognition than unrecognized BDI (3.2 vs 1.9, recognized vs unrecognized BDI, $\mathrm{P}<0.0001$ ) (Figure 1).

To understand the analysis of linguistic qualifiers, one must remember that among cases with intra-operative injury detection, the operative report was dictated with knowledge of the event, so linguistic qualifiers in these cases should reflect increased uncertainty. This was the case: qualifiers of uncertainty were present in $47 \%$ of BDI cases recognized 
during surgery. In unrecognized BDI cases, only $10 \%$ had qualifiers of uncertainty. But among the non-injury cases, only $2 \%$ used language indicating uncertainty $(\mathrm{P}=0.04$, compared to unrecognized $\mathrm{BDI})$.

Qualifiers of certainty during cystic duct dissection, however, were not different among the groups. Recognized and unrecognized BDI reflected the same level of certainty (18\% vs 19\% respectively), and while certainty was a little more common in uncomplicated cases $(23 \%)$, this was not statistically different. The overall distribution of linguistic qualifiers (Figure 2) was different among the three groups.
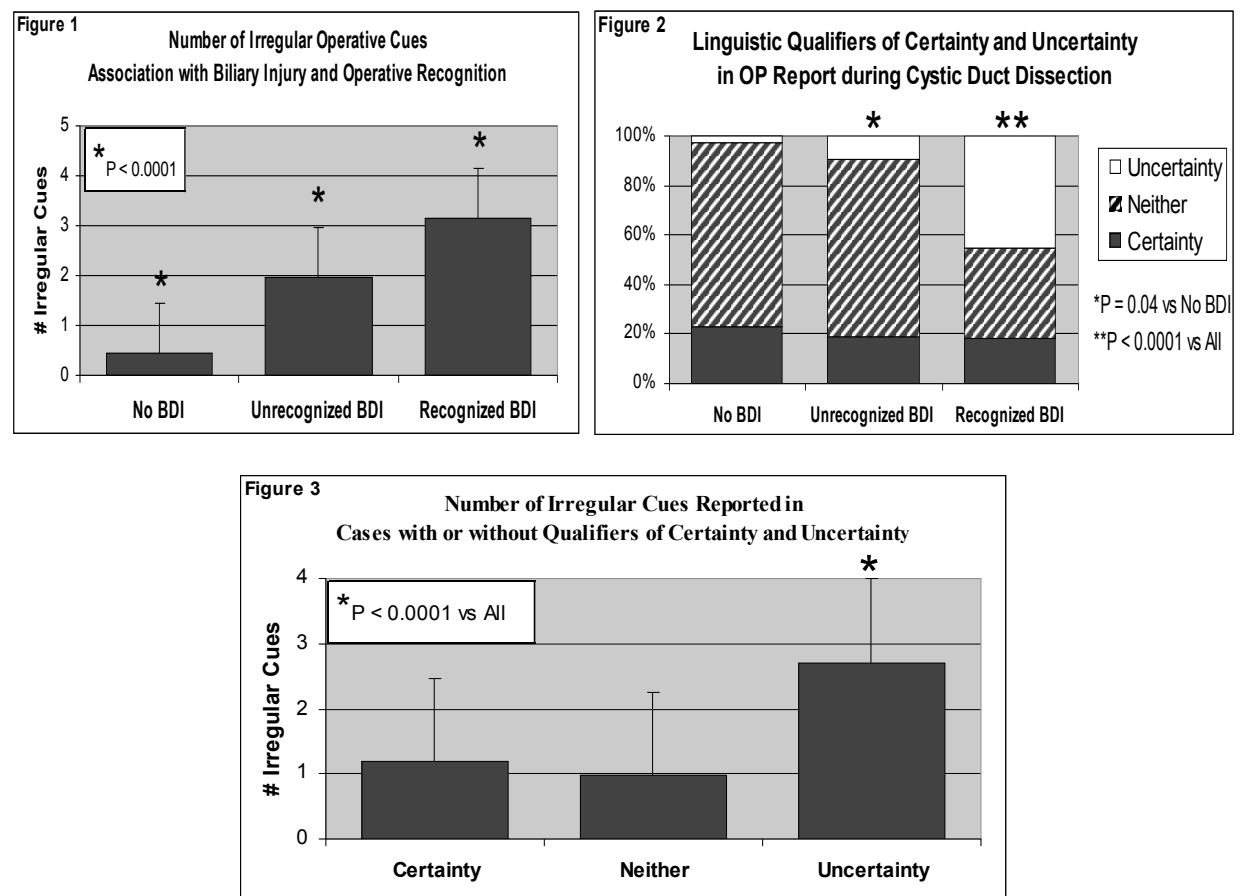

An increased number of irregular cues was reported in operative reports with qualifiers of uncertainty (Figure 3), but there were no differences between cases with no qualifiers and those with qualifiers of certainty. The correlation between irregular cues and uncertainty-language makes sense, in that surgeons who noted irregular cues would be more likely to express uncertainty about the case, while those who did not would have little basis for uncertainty.

\section{CONCLUSION}

These data provide insights into the surgeons' intra-operative thought processes. Irregular cues were more commonly reported by surgeons in cases where BDI were recognized intra-operatively, and they were uncommon in uncomplicated cases. This suggests that an accumulation of irregular cues may lead to re-framing of the surgeons' situation assessment. Also, the surgeon's unconscious use of qualifiers of certainty or uncertainty gave meaningful clues to their perspective of the operation. Qualifiers of uncertainty were more commonly associated with complicated cases, even when the injury was not recognized intra-op, suggesting that the choice of words reflected an unconscious uneasiness about the procedure. And, among BDI cases recognized intra-op, qualifiers of uncertainty were much more common. This supports the suspicion that these qualifiers reflected the surgeon's perspective. In contrast, qualifiers of certainty did not correlate with BDI recognition or an uncomplicated case. This is consistent with our previous observation that surgeons completing these procedures are most often confident that the procedure was uncomplicated. That certainty is not increased in uncomplicated cases, or decreased in complicated cases, underscores the compelling nature of the visual illusion associated with the laparoscopic environment that facilitates these injuries.

\section{REFERENCES}

Way, L.W., Stewart, L., Gantert, W., Liu, K., Lee, C.M., Whang, K., Hunter, J.G. (2003) Causes and Prevention of Laparoscopic Bile Duct Injuries: An Analysis of 252 Cases from a Human Factors and Cognitive Psychology Perspective. Ann Surg 237: 460-469.

Stewart, L. \& Way, L.W. (2007) The Prevention of Laparoscopic Bile Duct Injuries: an Analysis of 300 Cases of from a Human Factors and Cognitive Psychology Perspective. Proceedings of the Human Factors and Ergonomics Society 51st Annual Meeting,

Stewart, L., Dominguez, C.O., Way, L.W. (2007) Bile Duct Injuries in Laparoscopic Cholecystectomy: a Sensemaking Analysis of Operative Reports. K Mosier \& U Fischer (Eds.), Proceedings of the 8th International Conference on Naturalistic Decision making. 